\title{
Low density lipoprotein quality and discordance with apolipoprotein B in intensively controlled Type 1 diabetes: Any relationship with nutrition?
}

\author{
R.J. Webb ${ }^{1}$, I.G. Davies ${ }^{1}$, T.S. Purewal ${ }^{2}$, P.J. Weston ${ }^{2}$, G. Morrison ${ }^{2}$ and J.C. Abayomi ${ }^{1}$ \\ ${ }^{1}$ Food, Nutrition and Health Research Group, Liverpool John Moores University, Barkhill Road, Aigburth, Liverpool, \\ Merseyside, $L 17$ 6BD and \\ ${ }^{2}$ Department of Endocrinology and Diabetes, Royal Liverpool and Broadgreen University Hospital, Prescot Street, \\ Liverpool, Merseyside, L7 $8 X P$
}

Type 1 diabetes (T1D) is partly characterised by a higher prevalence of cardiovascular disease (CVD). Despite low density lipoprotein cholesterol (LDL-C) being a commonly treated target, apolipoprotein B (Apo B) has been shown to be a superior predictor of CVD and discordance between these two markers may predispose patients to altered risk ${ }^{(1)}$. The distribution of LDL-C also contributes to these risks, with LDL III \& IV fractions possessing greater atherogenic potential ${ }^{(2)}$. Few studies have investigated LDL-C quality and it's discordance with Apo B in relation to the nutritional intake of patients with intensively controlled Type 1 diabetes. The aim of this study was to address this dearth of research.

Following ethical approval and informed consent 28 patients (32\% male; $68 \%$ female) (mean age $48 \pm 15)$ were asked to complete a food frequency questionnaire (FFQ), donate a sample of blood and allow the authors access to their medical records to determine $\mathrm{HbA}_{1 \mathrm{c}}$. The initial FFQ responses were processed using FETA software. The blood sample was analysed for LDL-C, constituent subfractions and Apo B. All data were interrogated using descriptive statistics. Dichotomous dependent variables pertaining to LDL-C and Apo B were compared using McNemar's test and correlations between dietary variables were determined with Spearman's rho test.

Significant differences were shown between LDL-C categories when compared to Apo B $(p=0.039)$ and the majority of patients $(46.4 \%$ ) presented LDL-C $>2.0 \mathrm{mmol} / \mathrm{L}$ and Apo B $>80 \mathrm{mg} / \mathrm{dL}$ (Fig. 1). Although not discordant, these findings still suggest an increased risk according to recommendations ${ }^{(3)}$. Closer inspection of results revealed that individuals with raised LDL-C typically had an abundance of LDL I \& II fractions which may somewhat reduce this risk (Fig. 2). Spearman's correlation applied to the whole population produced no relationship between diet and LDL-C or Apo B; however, when focussing on the predominant 'at risk' cluster significant and strong relationships between LDL-C and total carbohydrate $\left(R^{2}=0 \cdot 835 ; p=<0 \cdot 001\right)$ and sucrose $\left(R^{2}=0.758 ; p=0.003\right)$ were found. No hypoglycaemia data were collected and the authors tentatively speculate that these relationships may be a consequence of its treatment. In the light of the small sample size a further more comprehensive investigation with an appropriately powered sample would be beneficial.

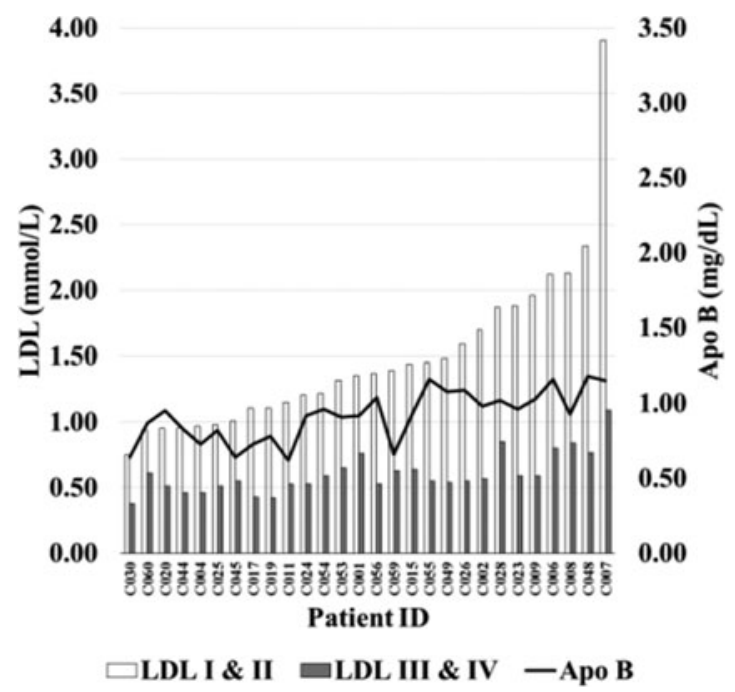

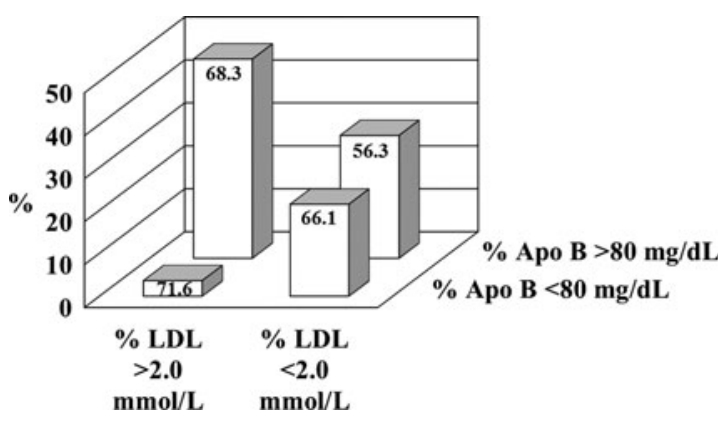

Fig. 2. Individual patient LDL subfractions d Apo B.

Fig. 1. Patient LDL-C and Apo B in relation to recommendations (Cluster $\mathrm{HbAl}_{\mathrm{c}}(\mathrm{mmol} / \mathrm{mol})$ shown on columns).

1. Otvos J et al. (2011) J Clin Lipidol 5, 105-113.

2. Vergès B (2009) Diabetes \& Metabolism 35, 353-60.

3. Catapano AL et al. (2016) Eur Heart J 37, 2999-3058. 